\title{
The value of KRAS mutation testing with CEA for the diagnosis of pancreatic mucinous cysts
}

Authors

Institutions
Abdurrahman Kadayifci ${ }^{1,2}$, Mohammad Al-Haddad ${ }^{3,4}$, Mustafa Atar ${ }^{1}$, John M. Dewitt ${ }^{3}$, David G. Forcione', Stuart Sherman ${ }^{3}$, Brenna W. Casey ${ }^{1}$, Carlos Fernandez-del Castillo ${ }^{5}$, C. Max Schmidt ${ }^{6}$, Martha B. Pitman', William R. Brugge ${ }^{1}$

Institutions are listed at the end of article. submitted:

25. September 2015 accepted after revision 18. January 2016

\section{Bibliography}

DOI http://dx.doi.org/

10.1055/s-0042-101755

Published online: 24.3.2016

Endoscopy International Open

2016; 04: E391-E396

(c) Georg Thieme Verlag KG

Stuttgart · New York

E-ISSN 2196-9736

\section{Corresponding author}

\section{Dr. Abdurrahman Kadayifci}

3-H GI Associates, Zero Emerson

Place,

Blossom st. Massachusetts

General Hospital,

Boston, 02114, MA

Fax: +1- 617-724-5997

akadayifci@mgh.harvard.edu

kadayifci@hotmail.com
Background and aims: Pancreatic cyst fluid (PCF) CEA has been shown to be the most accurate preoperative test for detection of cystic mucinous neoplasms (CMNs). This study aimed to assess the added value of PCF KRAS mutational analysis to CEA for diagnosis of CMNs.

Patients and methods: This is a retrospective study of prospectively collected endoscopic ultrasonography (EUS) fine-needle aspiration (FNA) data. KRAS mutation was determined by direct sequencing or equivalent methods. Cysts were classified histologically (surgical cohort) or by clinical (EUS or FNA) findings (clinical cohort). Performance characteristics of KRAS, CEA and their combination for detection of a cystic mucinous neoplasm (CMN) and malignancy were calculated. Results: The study cohort consisted of 943 patients: 147 in the surgical cohort and 796 in the clinical cohort. Overall, KRAS and CEA each had

\section{Introduction}

$\nabla$

Pancreatic cystic lesions (PCLs) are divided into two main classes, cystic mucinous neoplasms (CMNs) and non-mucinous cysts. CMNs consist of intraductal papillary mucinous neoplasms (IPMNs) and mucinous cystic neoplasm (MCNs). Mucinous and non-mucinous classification is extremely important since these cysts are premalignant neoplasms with potential for progression to pancreatic cancer.

Cross-sectional imaging methods and particularly endoscopic ultrasonography (EUS) have a significant role in diagnosis and differentiation of PCLs $[1,2]$. However, there are some limitations to imaging alone and imaging may not be helpful in differentiating between various types of cysts. EUS fine-needle aspiration (EUS-FNA) of cystic lesions allows for biochemical and cytological analysis of cyst fluid that might be further helpful for differentiation [2,3]. Among all cyst fluid diagnostic parameters, pancreatic cyst fluid (PCF) carci- high specificity (100\% and $93.2 \%$ ), but low sensitivity $(48.3 \%$ and $56.3 \%$ ) for the diagnosis of a CMN. The positivity of KRAS or CEA increased the diagnostic accuracy (80.8\%) and AUC (0.84) significantly compared to KRAS (65.3\% and 0.74 ) or CEA (65.8\% and 0.74$)$ alone, but only in the clinical cohort $(P<0.0001$ for both). KRAS mutation was significantly more frequent in malignant CMNs compared to histologically confirmed nonmalignant $\mathrm{CMNs}$ ( $73 \%$ vs. $37 \%, P=0.001$ ). The negative predictive value of KRAS mutation was $77.6 \%$ in differentiating non-malignant cysts.

Conclusions: The detection of a KRAS mutation in PCF is a highly specific test for mucinous cysts. It outperforms CEA for sensitivity in mucinous cyst diagnosis, but the data does not support its routine use.

noembryonic antigen (CEA) concentration alone has been suggested as the most accurate test for the diagnosis of CMNs at a cut-off level of $192 \mathrm{ng} /$ $\mathrm{mL}$. However, the diagnostic sensitivity of PCF CEA at a cut-off level of $192 \mathrm{ng} / \mathrm{mL}$ has been reported to be less than $60 \%$ in recent clinical series [4-6].

The presence of KRAS mutation in PCF in recent studies has been suggested as a highly specific test for the classification of a cyst as mucinous [7-9]. However, the number of patients included in some studies was small, with a limited number of studies using histology as the diagnostic 'reference standard [8-11]. Therefore, based on current reports, it is not certain whether routine testing for KRAS mutations in PCF is warranted. Our study primarily aimed to assess the value of KRAS mutational analysis along with CEA in PCF for accurate diagnosis of mucinous cysts. The study also sought to assess the value of KRAS mutation analysis in the differentiation of malignant CMNs. 


\section{Patients and methods}

$\nabla$

The study is a retrospective analysis of prospectively collected EUS-FNA data from two academic medical centers, Massachusetts General Hospital, (MGH) and Indiana University (IU). Patients gave their consent for FNA of the cyst and for fluid analysis, and a cyst registry was approved by the Institutional Review Boards (IRB) at both institutions. Consecutive patients who underwent an EUS-FNA for PCLs between 2006-2014 and who had sufficient fluid for KRAS mutation analysis were enrolled in the study. Exclusion criteria included patients with a bleeding tendency (INR $>1.5$, partial thromboplastin time $>50$ sec or platelets $<50,000 \mathrm{~mm}^{3}$ ), a solid mass, a history of pancreatic cancer, acute pancreatitis, a high clinical suspicion of a pseudocyst or pancreatic abscess, pancreatic ductal adenocarcinoma with cystic degeneration, cysts of extra-pancreatic origin and fluid provided by FNA of the main pancreatic duct.

The study data were recorded in a prospectively maintained database and the patients were divided into two separate groups for all analysis according to their management. Patients who underwent a surgical resection were included in the surgical cohort, and patients who were managed non-surgically included in the clinical cohort.

\section{EUS-FNA}

Patients who had a PCL $\geq 10 \mathrm{~mm}$ on cross-sectional imaging, and who were referred for EUS-FNA made up the study population. The location, size, number and morphology (mural nodule, wall thickness, septations, adjacent mass, ductal communication) of cysts was recorded. EUS-FNA was attempted if the cyst diameter was $\geq 10 \mathrm{~mm}$ and if the results could potentially impact patient management. The PCF was triaged for cytological examination and biochemical (CEA and amylase) analysis first and then for molecular testing. KRAS mutation testing was performed selectively when the fluid volume was more than $0.5 \mathrm{~mL}$.

To allow comparison of accuracy with KRAS analysis, cyst fluid CEA level was not used as a reference to differentiate mucinous cysts in the clinical cohort. A cut-off CEA value of $192 \mathrm{ng} / \mathrm{mL}$ was considered positive for only diagnostic calculations.

\section{Pathology and cytology}

Cysts were classified by histological examination as either mucinous (main-duct (MD), branch-duct (BD) or combined (COM) [IPMN or MCN]) or non-mucinous cysts (serous, pseudocyst, neuroendocrine and others). IPMNs were further subclassified according to the degree of dysplasia using 2010 WHO criteria [12]; (i) IPMN with low- or intermediate-grade dysplasia, (ii) IPMN with high-grade dysplasia (HGD), and (iii) IPMN with an associated invasive carcinoma. Malignant cysts were defined as cysts with either HGD or invasive carcinoma.

Cytological analyses of PCF were reported as non-diagnostic (because of insufficient cells or contamination) or diagnostic (adequate for evaluation of cellular elements). Diagnostic samples were classified either as mucinous (thick, colloid-like extracellular mucin without cells, mucinous epithelium with cytoplasmic mucin, low-grade mucinous epithelium in a transduodenal FNA, high-grade atypia or adenocarcinoma) or non-mucinous [13]. Nonspecific gastric-foveolar type epithelium without thick extracellular mucin in the transgastric FNAs was defined as nondiagnostic.

\section{Classification of cysts}

The mucinous/non-mucinous cyst classification of cysts in the surgical cohort was based on surgical histology results. The cysts in the clinical cohort were classified as mucinous if they had one of the cytological criteria (thick, colloid-like extra-cellular mucin without cells, mucinous epithelium with cytoplasmic mucin, low-grade mucinous epithelium in a transduodenal FNA, highgrade atypia or adenocarcinoma) or one of the EUS criteria [14] (mural nodule, wall thickness, septations, adjacent mass, ductal communication). These criteria are summarized in $\bullet$ Table 1. The cysts without any cytological or EUS criteria for a mucinous cyst were classified as non-mucinous.

\section{KRAS analysis}

KRAS 2-point mutation analysis on codons 12 and 13 was determined by fluorescent-based direct sequencing (PathFinder $\mathrm{TG}^{\circledR}$; Redpath, Pittsburgh, PA, USA) for all cases from IU, and for cases from MGH until the year 2010 by using the method previously described [7]. Starting in 2010, KRAS analysis on codons 12 and 13 was performed by using a primer extension-based method (SNaPshot ${ }^{\circledR}$ Life Technologies) in the Molecular Pathology Department of MGH. PCF with no amplification or no mutation were accepted as KRAS mutation negative which represents wild-type KRAS.

\section{Surgery}

All patients with a clinical diagnosis of MCN, MD-IPMN, or COMIPMN were referred for surgical resection if they were good surgical candidates. Patients with BD-IPMNs were evaluated and referred to surgery according to International Consensus Guidelines from 2006 [15] and then 2012 [16].

\section{Data analysis}

The sensitivity, specificity, positive predictive value (PPV), negative predictive value (NPV) and diagnostic accuracy of KRAS, CEA and their combination for the diagnosis of a mucinous cyst were calculated. The true positivity and false positivity was accepted with the positivity of one of the tests. Receiver-operator curves were generated and the area under the curve (AUC) calculated for diagnostic value of the tests. AUC values of tests and their combinations were compared statistically. Comparisons between categorical variables were tested with Chi Square test. Independent Student $t$-test was used to compare mean values between two independent groups. The agreement between CEA and KRAS to differentiate mucinous cysts was measured by concordance correlation coefficient. An $r_{c}$ value of 1 represents perfect agreement and 0 represents the agreement expected from chance. A 2-tailed $P$ value $<0.05$ was considered statistically sig-

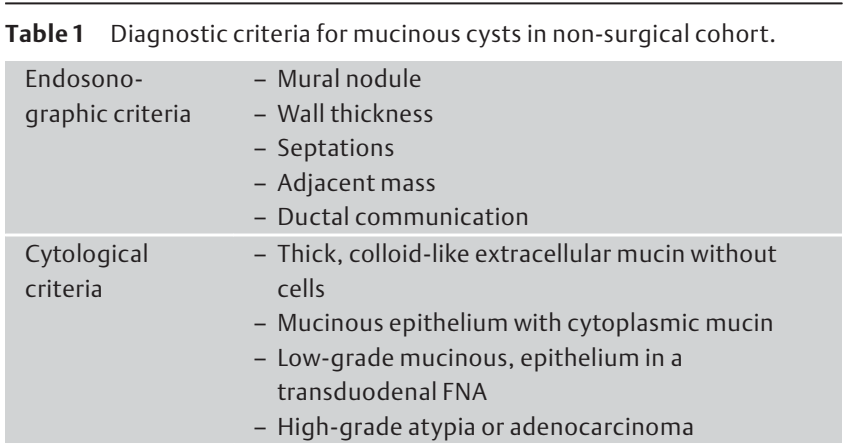

Abbreviation: FNA, fine-need aspiration 


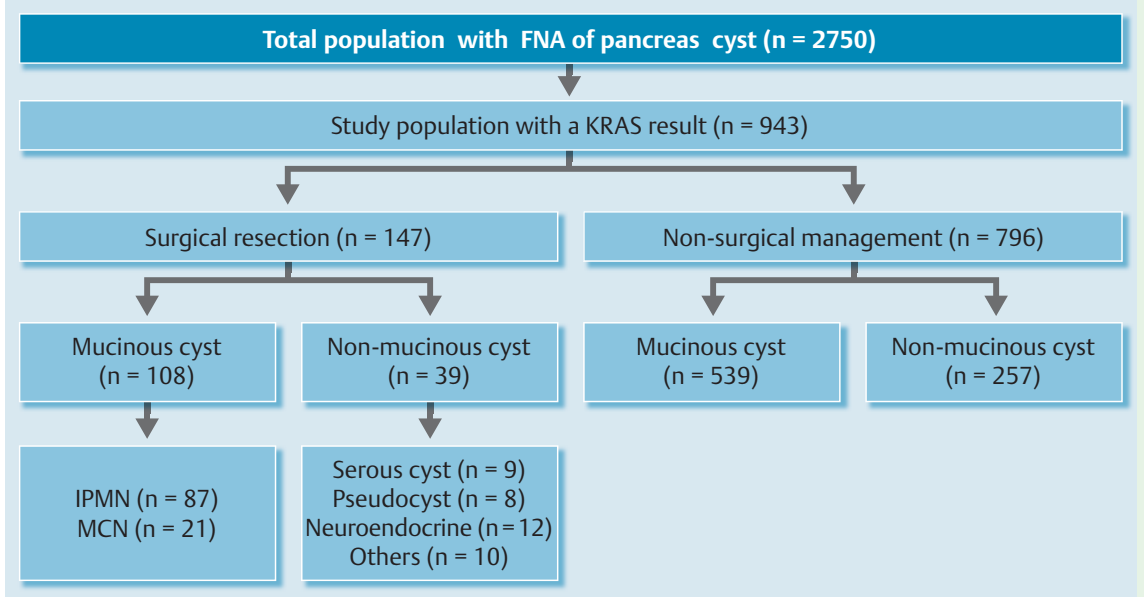

Fig. 1 Flowchart of the study population.

nificant. SPSS 15.0 package program (2006 SPSS Inc., Chicago, IL) was used for statistical analysis.

\section{Results}

$\nabla$

A total of 2750 patients underwent an EUS-FNA of a pancreatic cyst during the study period, of whom data on 943 with a KRAS mutation analysis result were available for analysis (the molecular analysis results of 282 cases from IU have been previously reported [17]). Patients were excluded from KRAS testing due to insufficient PCF volume, the gastroenterologist's decision not to test for KRAS mutation which was based on clinical findings, or due to technical failures of the test.

One hundred and forty-seven patients with a KRAS mutation analysis underwent surgical resection and made up the surgical cohort. The remaining 796 cases were classified by EUS findings or cytological results and they made up the clinical cohort. A flowchart of the study population is shown in $\bullet$ Fig. 1. Mucinous cysts constituted $73.4 \%(108 / 147)$ of patients in the surgical cohort and $67.7 \%$ (539/796) of patients in the clinical cohort. Cyst fluid CEA level $>192 \mathrm{mg} / \mathrm{dL}$ plays a key diagnostic role in mucinous cyst definition but we did not use it for the basic classification to avoid a flaw in the methods. However, the addition of CEA criteria to cytology plus EUS for a mucinous definition added 11 more cases to the mucinous group in the clinical cohort. These changes did not affect the overall performance of KRAS or KRAS + CEA in this cohort.

Baseline demographics, clinical and cyst characteristics of the study cohorts, based on mucinous classification, are provided in - Table 2

\section{Surgical pathology and cytology}

Mucinous cysts were malignant in 27 (invasive carcinoma in 19, and high-grade dysplasia in 8) of the patients based on the surgical pathology. Malignancy was identified in three cases of MCN. Cytological evaluation was non-diagnostic in 201 (25.2\%) of the clinical cohort because of inadequate or acellular material. A mucinous cyst was defined in 26\% (207/796) of the clinical cohort by cytology (adenocarcinoma in 15, high-grade atypia in 14, and by other cytological criteria in 178 cases) and $41.7 \%$ (332/796) by EUS criteria. Two hundred and fifty-seven patients in the clinical cohort (32.3\%) had none of the cytological or EUS criteria for a mucinous cyst, and their disease was classified as non-mucinous.

\section{KRAS and CEA diagnostic results}

The sensitivity, specificity, PPV, NPV, diagnostic accuracy and AUC value of KRAS positivity, CEA elevation and their combination for the diagnosis of a mucinous cyst as a whole and for each cohort are provided in Table 3. The PCF CEA level at a $192 \mathrm{ng} /$ $\mathrm{mL}$ cut-off value was highly specific at $93.2 \%$ but the sensitivity was found to be $56.3 \%$ in the whole group. The sensitivity of PCF CEA for a mucinous cyst diagnosis was lower in the clinical cohort than in the surgical cohort ( $54.6 \%$ vs. $63.2 \%, P=0.03$ ). The sensitivity of KRAS for a mucinous cyst diagnosis was $46.3 \%$ (50/108) and $48.8 \%$ (263/539) for the surgical and clinical cohorts, respectively, and the specificity was $100 \%$ for both. Adding the KRAS result to the CEA result increased the sensitivity to $13.8 \%$ in the surgical cohort and $21 \%$ in the clinical cohort. The diagnostic accuracy of the combined tests was more than $80 \%$ in all cohorts. The increased diagnostic accuracy and AUC with the KRAS-CEA combination was statistically significant compared to KRAS and CEA alone in the clinical cohort. The study parameters and assay results based on histological subtypes are shown in Table4. The KRAS positivity rate was higher in patients with IPMN than in those with MCN but the results were not statistically significant ( $49.4 \%$ vs. $33.3 \%, P=0.27$ ). KRAS mutation was positive in 12 patients in the surgical cohort and in 74 patients in the clinical cohort when CEA level is under $192 \mathrm{mg} / \mathrm{dL}$. The correlation coefficient rc was 0.23 for the surgical and 0.28 for the clinical cohorts, which demonstrated a weak correlation between KRAS and CEA results. The KRAS positivity and CEA levels did not show any correlation with the size, number and location of the cysts.

\section{KRAS and CEA in malignant cysts}

Based on histology and cytology, there were 56 patients with a malignant cyst (34 patients with adenocarcinoma and 22 patients with high-grade dysplasia/atypia) in the study population. The surgical cohort had 19 patients with adenocarcinoma and 8 patients with high-grade dysplasia. The clinical cohort had 15 patients with adenocarcinoma and 14 patients with high-grade atypia. KRAS mutation was positive in $73.2 \%(41 / 56)$ of malignant cysts. However, KRAS mutations were found in $37.3 \%$ (31/83) of surgically confirmed benign mucinous cysts. KRAS mutations were significantly more frequent in malignant mucinous cysts compared to benign mucinous cysts ( $73.2 \%$ vs. $37.3 \%, P=0.001$ ). Having a KRAS mutation in a mucinous cyst increased the relative risk of a malignant cyst to $1.96(1.42<\mathrm{RR}<2.70)$ and odds ratio to $5.58(2.06<0 R<10.32)$. In malignant cysts, KRAS mutation rate was higher in patients with adenocarcinoma (28/34, 82.3\%) 
Table 2 Demographic, clinical and cyst characteristics of the study cohorts based on the mucinous classification.

\begin{tabular}{|c|c|c|c|c|c|c|c|}
\hline & \multirow[b]{2}{*}{ Total } & \multicolumn{3}{|c|}{ Surgical cohort } & \multicolumn{3}{|c|}{ Clinical cohort } \\
\hline & & Mucinous & Non-mucinous & $p$ & Mucinous & $\begin{array}{l}\text { Non-muci- } \\
\text { nous }\end{array}$ & $p$ \\
\hline Number (n) & 943 & 108 & 39 & & 539 & 257 & \\
\hline Mean age (range), years & $\begin{array}{l}66.4 \\
(17-95)\end{array}$ & $\begin{array}{l}61.9 \\
(20-89)\end{array}$ & $\begin{array}{l}56.4 \\
(27-77)\end{array}$ & $P=0.053$ & $\begin{array}{l}70.3 \\
(17-95)\end{array}$ & $\begin{array}{l}61.2 \\
(23-88)\end{array}$ & $P=0.0001$ \\
\hline Gender, male/female & $384 / 559$ & $36 / 72$ & $17 / 22$ & $P=0.68$ & $222 / 317$ & $109 / 148$ & $P=0.8$ \\
\hline Symptomatic ${ }^{1}, \mathbf{n}(\%)$ & $524(56.2)$ & $70(64.8)$ & $21(53.8)$ & $P=0.42$ & $296(54.9)$ & $143(55.6)$ & $P=0.9$ \\
\hline Amylase > $250 \mathrm{mg} / \mathrm{dL},(\%)$ & 68.2 & 55.7 & 53.1 & $P=0.97$ & 74.5 & 63.7 & $P=0.02$ \\
\hline Cyst size (mean diameter \pm SD), $\mathrm{mm}$ & $25.3 \pm 16$ & $27 \pm 15$ & $23 \pm 14$ & $P=0.45$ & $24 \pm 17$ & $22 \pm 15$ & $P=0.13$ \\
\hline \multicolumn{8}{|l|}{ Cyst location (\%) } \\
\hline Head/neck/Unc. & 55 & 40 & 32 & & 58 & 55 & \\
\hline Body & 24 & 24 & 21 & $P=0.69$ & 25 & 23 & $P=0.48$ \\
\hline Tail & 21 & 36 & 47 & & 17 & 22 & \\
\hline \multicolumn{8}{|l|}{ Cyst number (\%) } \\
\hline Single & 69 & 70 & 75 & $P=0.95$ & 66 & 74 & $P=0.09$ \\
\hline Multiple & 31 & 30 & 25 & & 34 & 26 & \\
\hline
\end{tabular}

${ }^{1}$ One of the symptoms referable to the pancreas; abdominal pain, weight loss, obstructive jaundice, pancreatitis.

Table 3 Diagnostic value of CEA and KRAS to differentiate mucinous vs. non-mucinous cysts.

\begin{tabular}{|c|c|c|c|c|c|c|c|c|c|}
\hline \multirow[b]{2}{*}{ Test } & \multicolumn{3}{|c|}{ All patients $n=943$} & \multicolumn{3}{|c|}{ Surgical cohort n=147 } & \multicolumn{3}{|c|}{ Clinical cohort n=796 } \\
\hline & KRAS+ & CEA $\uparrow$ & $\begin{array}{l}\text { KRAS+ or } \\
\text { CEA } \uparrow\end{array}$ & KRAS+ & CEA $\uparrow$ & $\begin{array}{l}\text { KRAS+ or } \\
\text { CEA } \uparrow\end{array}$ & KRAS+ & CEA $\uparrow$ & $\begin{array}{l}\text { KRAS+ or } \\
\text { CEA } \uparrow\end{array}$ \\
\hline Sensitivity \% & 48.3 & 56.3 & 75.8 & 46.3 & 63.2 & 77 & 48.8 & 54.6 & 75.6 \\
\hline Specificity \% & 100 & 93.2 & 93.2 & 100 & 90.9 & 91.1 & 100 & 93.8 & 93.8 \\
\hline PPV \% & 100 & 95.4 & 96.6 & 100 & 94.8 & 95.7 & 100 & 95.6 & 96.8 \\
\hline NPV \% & 47 & 45.9 & 60.6 & 40.2 & 48.3 & 60.7 & 48.2 & 45.2 & 60.5 \\
\hline Accuracy \% & 64.5 & 66.8 & $80.9^{*}$ & 60.5 & 70.8 & $81.6^{\wedge}$ & 65.3 & 65.8 & $80.8^{1}$ \\
\hline AUC & 0.742 & 0.748 & $0.846^{*}$ & 0.730 & 0.781 & $0.838^{\sim}$ & 0.744 & 0.742 & $0.847^{1}$ \\
\hline
\end{tabular}

Abbreviation: PPV, positive predictive value; NPV, negative predictive value; AUC, area under curve

$1 P<0.0001$ vs. KRAS + and CEA $\uparrow$ alone, $\wedge p<0.01$ vs KRAS,$+>0.05$ vs CEA $\uparrow$ alone, $\sim p>0.05$ vs. KRAS + and CEA $\uparrow$ alone

Table 4 Study parameters based on histological cyst type $(n=147)$.

\begin{tabular}{|lllllll|}
\hline Parameters & IPMN & MCN & Serous & Pseudocyst & NET & Others \\
\hline Number (n) & 87 & 21 & 9 & 8 & 12 & 10 \\
\hline Mean age (range), years & $66.3(29-89)$ & $45.3(20-70)$ & $51.8(27-75)$ & $57.8(30-66)$ & $62.2(40-77)$ & $53.1(28-70)$ \\
\hline Gender, male/female & $35 / 52$ & $1 / 20$ & $3 / 6$ & $4 / 4$ & $8 / 4$ & $2 / 8$ \\
\hline KRAS (+), \% & 49.4 & 33.3 & 0 & 0 & 0 & 0 \\
\hline CEA $>$ 192 mg/dL, (\%) & 61.9 & 68.4 & 0 & 12.5 & 16.6 & 10 \\
\hline KRAS (+) or CEA $\uparrow(\%)$ & 77 & 73.6 & 0 & 12.5 & 16.6 & 10 \\
\hline Amylase $>\mathbf{2 5 0} \mathbf{m g} / \mathbf{d L},(\%)$ & 53.7 & 64.7 & 37.5 & 75 & 25 & 77 \\
\hline
\end{tabular}

Abbreviations: IPMN, intraductal papillary neoplasms; MCN, mucinous cystic neoplasm; NET, neuroendocrine tumor

than patients with high-grade dysplasia/atypia (13/22, 59\%). The study parameters between malignant cysts and surgically confirmed benign cysts are shown in 0 Table 5. Elevated PCF CEA level was found more frequently in malignant mucinous cysts compared to benign cysts ( $78.5 \%$ vs. $57.8 \%, P=0.02)$. The median CEA values of malignant cysts were also significantly higher than non-malignant cysts ( $921 \mathrm{mg} / \mathrm{dL}$ vs. $491 \mathrm{mg} / \mathrm{dL}, P=0.04$ ).

The combination of KRAS and CEA revealed $91 \%$ sensitivity but $30.1 \%$ specificity for a malignant cyst diagnosis. The NPV of KRAS mutation alone and together with CEA elevation was 77.6 $\%$ and $83.3 \%$, respectively, in differentiating non-malignant from malignant CMNs.

\section{Discussion}

The accurate diagnosis of PCLs is a key challenging issue for optimal clinical management. The high specificity of KRAS mutation for mucinous cysts has been confirmed in subsequent clinical studies but low sensitivity limited its widespread usage in routine PCF analysis $[10,18]$.

To our knowledge, the current study includes the largest cohort of KRAS mutation testing for CMN diagnosis. The results of our study confirmed high specificity of KRAS mutation for MCNs diagnosis in both surgical and clinical cohorts. However, the sensitivity of KRAS mutation alone was limited. The PCF CEA (cutoff $192 \mathrm{ng} / \mathrm{mL}$ ) was also highly specific for mucinous cysts but the 


\begin{tabular}{|llll|}
\hline Parameters & $\begin{array}{l}\text { Malignant mucinous cysts } \\
\text { (surgical pathology or cytology) }\end{array}$ & $\begin{array}{l}\text { Non-malignant mucinous cysts } \\
\text { (surgical pathology) }\end{array}$ & $\boldsymbol{P}$ \\
\hline Number (n) & 56 & 83 & \\
\hline Mean age (range), years & $73.1(44-91)$ & $62.3(20-89)$ & $P=0.002$ \\
\hline Gender, male/female & $20 / 36$ & $24 / 59$ & $P=0.6$ \\
\hline KRAS (+), \% & 73.2 & 37.3 & $P=0.001$ \\
\hline CEA (median, mg/dL) & $921(4.5-168.140)$ & $491(0.3-48.378)$ & $P=0.04$ \\
\hline CEA > 192 mg/dl, (\%) & 78.5 & 57.8 & $P=0.02$ \\
\hline KRAS (+) or CEA $\uparrow(\%)$ & 91 & 69.8 & $P=0.01$ \\
\hline Amylase $>\mathbf{2 5 0} \mathbf{m g} / \mathbf{d L},(\%)$ & 29.8 & 59.4 & $P=0.01$ \\
\hline
\end{tabular}

Table 5 Study parameters in patients with malignant vs. nonmalignant mucinous cysts. sensitivity was found to be lower than previously reported by the Cooperative Study [14]. The sensitivity of CEA for mucinous cysts diagnosis in the surgical cohort was higher than in the clinical cohort in our study. This might be a result of a selection bias of patients for surgery including malignant mucinous cysts and MCNs. A meta-analysis of 12 published studies showed that the pooled sensitivity and specificity of CEA for the differentiation between mucinous and non-mucinous cystic lesions was $63 \%$ and $88 \%$, respectively [19]. Most of these studies included surgically resected lesions because there is no optimal diagnostic standard for diagnosis of mucinous cyst in a clinical setting. As a result, these published series usually consisted of patients with malignant IPMNs, MCNs and BD-IPMNs with high-risk stigmata or worrisome features. In our study, we have found that CEA elevation was significantly more common in malignant mucinous cysts compared to non-malignant mucinous cysts.

The additive value of KRAS mutation testing to PCF CEA may have potential to increase the sensitivity for diagnosis of mucinous cysts. The combination of elevated CEA and KRAS mutation detected an additional 11 mucinous cysts in 76 cases in the PANDA study [7]. In another study, the sensitivity of KRAS mutation and elevated CEA for mucinous differentiation were $54 \%$ and $62 \%$, respectively, and the combination of both improved the sensitivity to $83 \%$ and maintained a high specificity of $85 \%$ [10]. In our study, the addition of KRAS analysis to CEA increased the sensitivity and diagnostic accuracy of CEA significantly without decreasing specificity. The ROC analysis also showed significantly larger AUC values with the combination of tests. However, we could not demonstrate a statistical difference in diagnostic parameters with use of combination tests in the surgical cohort. In fact, sensitivity, diagnostic accuracy and AUC values of combined tests were very similar between the surgical and the clinical cohorts. There could be two reasons for the lack of a statistical significance in the surgical cohort. First, the sensitivity of CEA alone for diagnosis of a mucinous cyst was higher in the surgical cohort than in the clinical cohort, which may have limited the added benefit of KRAS analysis to the CEA testing. Second, the sample size was smaller in the surgical cohort than in the clinical cohort, which decreased the power of the analysis to detect a statistical significance.

To detect the malignant potential of pancreatic cysts accurately is the ultimate goal of any diagnostic test. KRAS mutation was shown as one of the earliest genetic alterations in the majority of pancreatic ductal adenocarcinomas but its role and presence in the development of malignant cyst lacks clarity. The role of cyst fluid KRAS mutation for assessment of malignancy was first studied in 11 patients with malignant cysts, and was reported as positive in 10 of these cases [20]. The number of DNA mutations was found to be higher in malignant cysts in the PANDA study but the presence of KRAS mutation alone did not significantly differ between premalignant and malignant cysts [7]. The high-amplitude KRAS mutation followed by allelic loss was highly specific for the presence of malignancy (96\%) but the sensitivity remained low (37\%). The presence of PCF KRAS mutation was associated with progression and development of malignancy in longterm follow-up of 63 patients in another study by the same group [21]. Our data showed a high frequency of KRAS mutation in malignant cysts. Unlike the PANDA study, both low- and high-clonality mutations of KRAS were accepted as positive in our analysis. This may explain the higher sensitivity and lower specificity of KRAS mutation in our study for malignant cyst detection. Another interesting finding in our study was that cysts with high-grade dysplasia/atypia had a higher KRAS mutation rate than did nonmalignant cysts but a lower rate than adenocarcinomas. The number of cases with high-grade dysplasia/atypia was too small to make a conclusion, but a possible role of KRAS mutation in adenocarcinoma sequencing seems likely. KRAS mutation may be one of the important tests predicting mucinous cyst behavior, but our data show that the diagnostic value of KRAS positivity alone for malignant cysts remains limited since it lacks specificity for malignant non-malignant differentiation. Nevertheless, high NPV might be helpful to exclude a malignant cyst in clinical practice. The combination of KRAS positivity with CEA does not add diagnostic accuracy for malignant cysts but sensitivity and NPV increased to $90.9 \%$ and $80.9 \%$, respectively. The integration of molecular pathology with cytology, imaging and fluid chemistry determined the diagnosis of malignancy with a $90.6 \%$ sensitivity, $83.3 \%$ specificity and $89.6 \%$ accuracy [22].

GNAS mutation has been detected in PCF samples of patients with IPMN but not in fluid samples of other PCLs in recent studies [23]. It has high specificity but a limited sensitivity for a diagnosis of IPMN. The combination of KRAS and GNAS mutation analyses improved the sensitivity of molecular testing in preliminary studies $[23,24]$; however, more data are still needed to demonstrate the benefit of a combination.

There are certain limitations to this study. Because surgical pathology was the reference standard for a mucinous cyst diagnosis, the results of the surgical cohort, no doubt, will be more valuable. Our study included a significant number of patients but the majority of them were in the clinical cohort in whom a final mucinous/non-mucinous classification was solely based on EUS and/ or cytological criteria. To avoid introducing bias to the methods, we did not use CEA level and KRAS mutation for cyst classification in the clinical cohort. The specificity of cytology for mucinous cyst diagnosis using the defined study criteria is very high; however, it underestimates the number of mucinous cysts due to low sensitivity [3]. The EUS criteria used to define mucinous cysts are useful in clinical practice but their specificity and sensitivity are also limited. Therefore some of the mucinous cysts may have been included in the non-mucinous group. 
On the other hand, the study was conducted in two tertiary referral high-volume centers where all EUS were performed by highly skilled endosonographers in the field. In clinical practice, only a small proportion of pancreas cysts patients undergo surgery, thus limiting the sample size for an adequate power analysis. For this reason, well-designed studies need to include patients with a non-surgical diagnosis. Besides, the results of selected patients for surgery may be different than the unselected clinical cohort, and surgical results may not be applicable to all patients with pancreatic cysts. Therefore, we believe the sample size of our clinical cohort is sufficient to make a conclusion about the utilization of KRAS analysis.

Another limitation of this study was that the KRAS testing was not studied in all cysts but on a selected basis. This may limit the applicability of our results for all pancreatic cysts detected in EUS. The retrospective analysis of the outcomes data from the study databases was another drawback of the study. In spite of the prospective collection of data, some missing or insufficient data could undermine the study objectives. The analysis of KRAS mutation by two different methods is another potential weakness of the study. However, the comparison of the SNaPshot genotyping system with direct DNA sequencing showed $100 \%$ consistency in the validation study [25]. Performance characteristics of different KRAS mutation analysis techniques including both methods were found to be highly concordant [26]. We do not think the use of KRAS analysis based on two different methods has an impact on our study results because both methods are reliable and were validated in different studies.

In summary, the detection of a KRAS mutation in PCF is a highly specific test for mucinous cysts. The addition of KRAS testing to CEA outperforms its sensitivity but does not improve the diagnostic accuracy in the surgical cohort. KRAS mutations are more frequently detected in malignant mucinous cysts than in nonmalignant cysts but the diagnostic accuracy is limited because of low specificity. In conclusion, our data do not support routine use of KRAS testing in traditional PCF analysis, but it might be potentially useful in selected cases.

\section{Competing interests: None}

Institutions

Division of Gastroenterology, Massachusetts General Hospital, Harvard

Medical School, Boston, Massachusetts, United States

2 Division of Gastroenterology, Gaziantep University School of Medicine, Gaziantep, Turkey

Division of Gastroenterology and Hepatology, Indiana University School of Medicine, Indianapolis, Indiana, United States

${ }^{4}$ Digestive Disease Center, Cleveland Clinic, Abu Dhabi, United Arab Emirates 5 Department of Surgery, Massachusetts General Hospital, Harvard Medical School, Boston, Massachusetts, United States

${ }^{6}$ Department of Surgery, Indiana University School of Medicine, Indianapolis, Indiana, United States

Department of Pathology, Massachusetts General Hospital, Harvard Medical School, Boston, Massachusetts, United States

\section{References}

1 Mortele KJ. Cystic pancreatic neoplasms: imaging features and management strategy. Semin Roentgenol 2013; 48: 253-263

2 Brugge WR. The use of EUS to diagnose cystic neoplasms of the pancreas. Gastrointest Endosc 2009; 69: 203-209

3 Pitman MB, Lewandrowski K, Shen J et al. Pancreatic cysts: preoperative diagnosis and clinical management. Cancer Cytopathol 2010; 118: 1 13
4 Rogart JN, Loren DE, Singu BS et al. Cyst wall puncture and aspiration during EUS-guided fine needle aspiration may increase the diagnostic yield of mucinous cysts of the pancreas. J Clin Gastroenterol 2011; 45: $164-169$

5 de Jong K, van Hooft JE, Nio CY et al. Accuracy of preoperative workup in a prospective series of surgically resected cystic pancreatic lesions. Scand J Gastroenterol 2012; 47: 1056-1063

6 Chai SM, Herba K, Kumarasinghe MP et al. Optimizing the multimodal approach to pancreatic cyst fluid diagnosis: developing a volumebased triage protocol. Cancer Cytopathol 2013; 121: 86-100

7 Khalid A, Zahid M, Finkelstein SD et al. Pancreatic cyst fluid DNA analysis in evaluating pancreatic cysts: a report of the PANDA study. Gastrointest Endosc 2009; 69: 1095-1102

8 Sawhney MS, Devarajan S, O'Farrel $P$ et al. Comparison of carcinoembryonic antigen and molecular analysis in pancreatic cyst fluid. Gastrointest Endosc 2009; 69: 1106-1110

9 Talar-Wojnarowska $R$, Pazurek M, Durko L et al. A comparative analysis of K-ras mutation and carcinoembryonic antigen in pancreatic cyst fluid. Pancreatology 2012; 12: 417-420

10 Nikiforova MN, Khalid A, Fasanella KE et al. Integration of KRAS testing in the diagnosis of pancreatic cystic lesions: a clinical experience of 618 pancreatic cysts. Mod Pathol 2013; 26: 1478-1487

11 Sreenarasimhaiah J, Lara LF, Jazrawi SF et al. A comparative analysis of pancreas cyst fluid CEA and histology with DNA mutational analysis in the detection of mucin producing or malignant cysts. JOP 2009; 10: $163-168$

12 Bosman F. WHO classification of tumours of the digestive system. Lyon, France: IARC Press; 2010

13 Pitman MB, Centeno BA, Daglilar ES et al. Cytological criteria of highgrade epithelial atypia in the cyst fluid of pancreatic intraductal papillary mucinous neoplasms. Cancer Cytopathol 2014; 122: 40-47

14 Brugge WR, Lewandrowski K, Lee-Lewandrowski $E$ et al. Diagnosis of pancreatic cystic neoplasms: a report of the cooperative pancreatic cyst study. Gastroenterology 2004; 126: 1330-1336

15 Tanaka M, Chari S, Adsay Vet al. International consensus guidelines for management of intraductal papillary mucinous neoplasms and mucinous cystic neoplasms of the pancreas. Pancreatology 2006; 6: 17 - 32

16 Tanaka M, Fernandez-del Castillo C, Adsay V et al. International consensus guidelines 2012 for the management of IPMN and MCN of the pancreas. Pancreatology 2012; 12: 183 - 197

17 Al-Haddad M, DeWitt J, Sherman S et al. Performance characteristics of molecular (DNA) analysis for the diagnosis of mucinous pancreatic cysts. Gastrointest Endosc 2014; 79: 79-87

18 Shen J, Brugge WR, Dimaio CJ et al. Molecular analysis of pancreatic cyst fluid: a comparative analysis with current practice of diagnosis. Cancer 2009; 117: $217-227$

19 Thornton GD, McPhail MJ, Nayagam $S$ et al. Endoscopic ultrasound guided fine needle aspiration for the diagnosis of pancreatic cystic neoplasms: a meta-analysis. Pancreatology 2013; 13: 48-57

20 Khalid A, McGrath KM, Zahid $M$ et al. The role of pancreatic cyst fluid molecular analysis in predicting cyst pathology. Clin Gastroenterol Hepatol 2005; 3: 967 - 973

21 Rockacy MJ, Zahid M, McGrath KM et al. Association between KRAS mutation, detected in pancreatic cyst fluid, and long-term outcomes of patients. Clin Gastroenterol Hepatol 2013; 11: 425-429

22 Al-Haddad MA, Kowalski T, Siddiqui A et al. Integrated molecular pathology accurately determines the malignant potential of pancreatic cysts. Endoscopy 2015; 47: 136-142

$23 \mathrm{Wu}$ J, Matthaei H, Maitra A et al. Recurrent GNAS mutations define an unexpected pathway for pancreatic cyst development. Sci Transl Med 2011; 3: 92ra66

24 Singhi $A D$, Nikiforova MN, Fasanella KE et al. Preoperative GNAS and KRAS testing in the diagnosis of pancreatic mucinous cysts. Clin Cancer Res 2014; 20: $4381-4389$

25 Magnin S, Viel E, Baraquin A et al. A multiplex SNaPshot assay as a rapid method for detecting KRAS and BRAF mutations in advanced colorectal cancers. J Mol Diagn 2011; 13: 485 - 492

26 Anderson SM. Laboratory methods for KRAS mutation analysis. Expert Rev Mol Diagn 2011; 11: 635-642 